\title{
Binary Step Size Variations of LMS and NLMS
}

\author{
C Mohan Rao ${ }^{1}$, Dr. B Stephen Charles ${ }^{2}$, Dr. M N Giri Prasad ${ }^{3}$ \\ ${ }^{1}$ Asso. Prof., Department of ECE, NBKRIST, Vidhyanagar, India \\ ${ }^{2}$ Principal, SSCET, Kurnool, India \\ ${ }^{3}$ Prof. \& Head, Department of ECE, JNTUACE, Pulivendula, India
}

\begin{abstract}
Due to its ease of implementation, the least mean square (LMS) algorithm is one of the most wellknown algorithms for mobile communication systems. However, the main limitation of this approach is its relatively slow convergence rate. In this paper two new variable step size Least Mean Square (LMS) adaptive filter algorithms are proposed. In the first algorithm two step sizes will be calculated from two values which will vary iteration to iteration. This algorithm is analogous to LMS algorithm, and produces better convergence performance compared to that of LMS. In the second algorithm also two step sizes are calculated based on a variable. This algorithm is analogous to Normalised Least Mean Square (NLMS) and produces better convergence performance compared to that of NLMS.
\end{abstract}

Keywords -LMS, NLMS, Binary Step, Channel Equalization.

\section{INTRODUCTION}

Consider the communication system depicted in the figure 1. The communication channel is continuous, but due to matched filtering, the effective signaling from the Transmitter pulse shaping to Receiver matched filter is discrete. Then, we can model the system as a discrete one.

$$
y[n]=\sum_{l=0}^{L-1} h_{l} x[n-l]+v[n]
$$

If $h_{l} \neq 0, l \neq 0$, the channel causes ISI. A symbol $x[n]$ interferes next $L-1$ symbols. Matched-filter alone cannot overcome this degradation. An equalizer is needed.

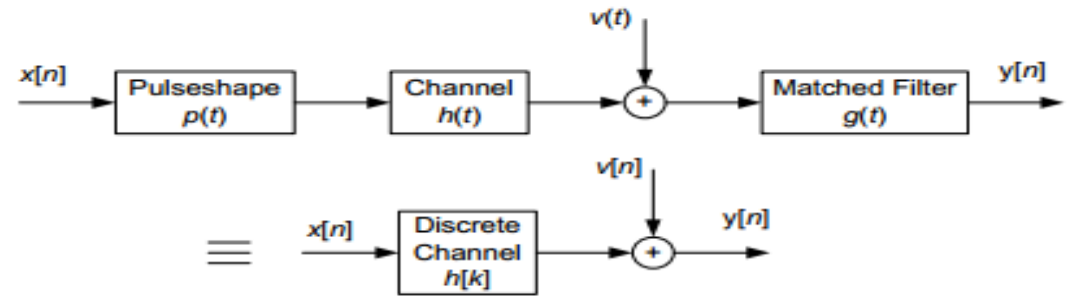

Figure 1. Communication System

An equalizer can be classified in several different ways as it was shown in figure 2.

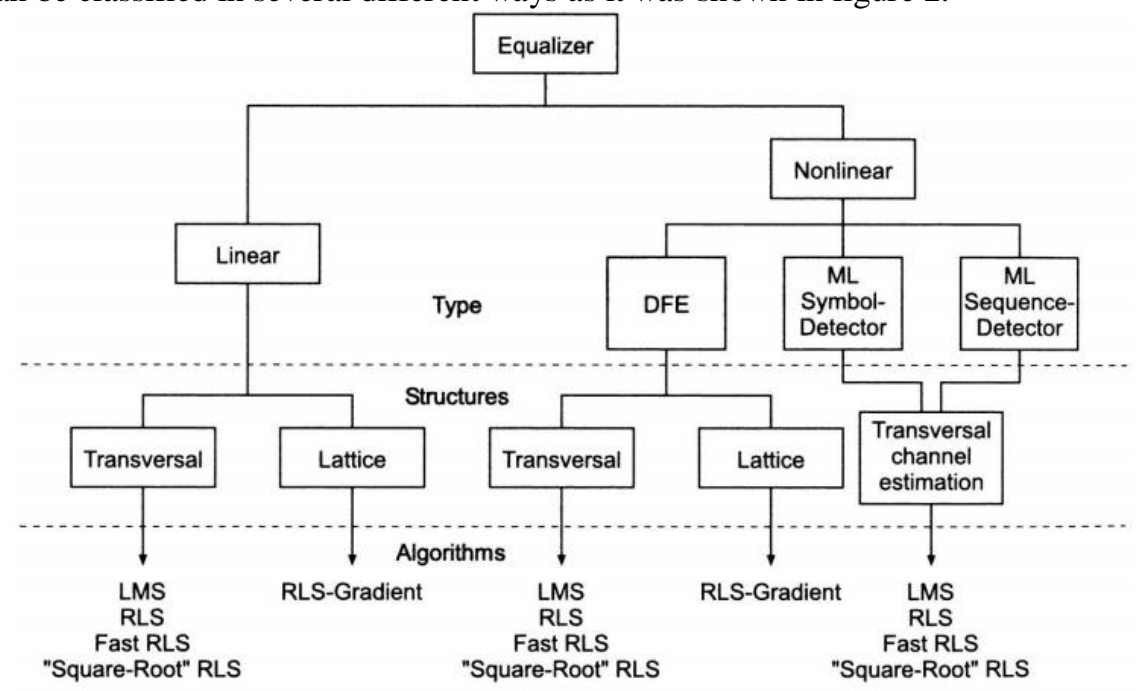

Figure 2. Types of Equalizers 


\subsection{Related Work}

In the field of adaptive signal processing, the least mean square (LMS) algorithm is an extensively explored algorithm due to its simplicity [1-3]. The LMS algorithm has been widely used in mobile communications $[4-6,7,8]$ such as multi-user detection, adaptive antenna arrays, and equalizations, to name just a few. The main limitation of the algorithm is its convergence rate $[6,10]$. That is, the LMS algorithm has the conflicting requirements of a small step-size parameter to reduce mis-adjustment and a large step-size parameter to achieve fast convergence. There are two main categories for methods addressing the convergence rate issue: time-domain and transform-domain methods.

In the time-domain category, researchers constantly look for new methods of step size selection to improve convergence rate. The most common scheme is the gear shifting approach [9], which uses a large step size during the transient state and then shifts to a smaller size during the steady state. Other important timedomain approaches include variable step-size LMS algorithms, which make the step size data-dependent. However, when the input signal is highly colored, LMS algorithms tend to produce a slow convergence rate. Previous methods alleviate the correlation of the input signal by pre-whitening it using a number of transforms, of which the frequency domain versions are more common. Frequency-domain LMS algorithms can increase the convergence speed for broadband signals.

In the current paper two variable step size LMS algorithms are proposed, in which one algorithm named Binary Step Size LMS outperforms LMS and other named Binary Step Size NLMS outperforms NLMS. The rest of the paper was organized as follows. In the next two sections LMS and NLMS algorithms are explained. In the section 4 , the binary step size algorithms are presented. The simulation results are given in the section 5 and the section 6 concludes this paper.

\section{LEAST MEAN SQUARES FILTER (LMS)}

Least mean squares (LMS) algorithms are a class of adaptive filter used to mimic a desired filter by finding the filter coefficients that relate to producing the least mean squares of the error signal (difference between the desired and the actual signal). It is a stochastic gradient descent method in that the filter is only adapted based on the error at the current time. It was invented in 1960 by Stanford University professor Bernard Widrow and his first Ph.D. student, Ted Hoff [11].

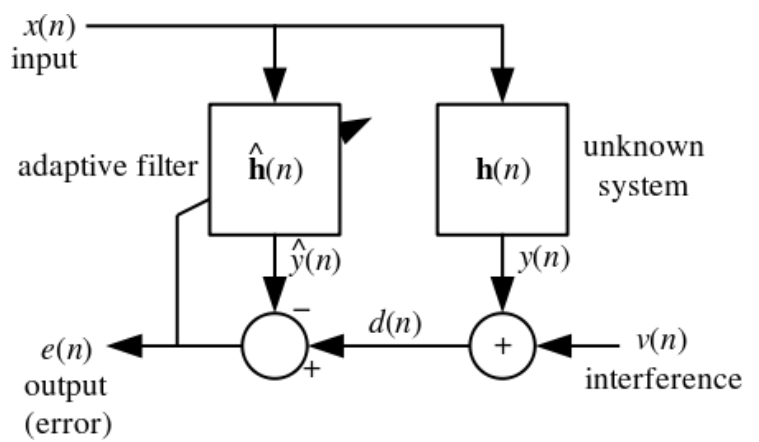

Figure 3. LMS Adaptive Filtering

\subsection{Relationship to the least mean squares filter}

The realization of the causal Wiener filter looks a lot like the solution to the least squares estimate, except in the signal processing domain. The least squares solution, for input matrix $\mathbf{X}$ and output vector $\mathbf{y}$ is

$$
\hat{\beta}=\left(X^{T} X\right)^{-1} X^{T} y .
$$

The FIR Wiener filter is related to the least mean squares filter, but minimizing its error criterion does not rely on cross-correlations or auto-correlations. Its solution converges to the Wiener filter solution. Most linear adaptive filtering problems can be formulated using the block diagram above. That is, an unknown system $h(n)$ is to be identified and the adaptive filter attempts to adapt the filter $\hat{h}(n)$ to make it as close as possible to $h(n)$, while using only observable signals $x(n), d(n)$ and $e(n)$; but $y(n), v(n)$ and $h(n)$ are not directly observable. Its solution is closely related to the Wiener filter.

The basic idea behind LMS filter is to approach the optimum filter weights $\left(R^{-1} P\right)$, by updating the filter weights in a manner to converge to the optimum filter weight. The algorithm starts by assuming a small weights (zero in most cases), and at each step, by finding the gradient of the mean square error, the weights are updated. That is, if the MSE-gradient is positive, it implies, the error would keep increasing positively, if the 
same weight is used for further iterations, which means we need to reduce the weights. In the same way, if the gradient is negative, we need to increase the weights. So, the basic weight update equation is :

$W_{n+1}=W_{n}-\mu \Delta \varepsilon[n]$, where $\mathcal{E}$ represents the mean-square error. The negative sign indicates that, we need to change the weights in a direction opposite to that of the gradient slope.

The mean-square error, as a function of filter weights is a quadratic function which means it has only one extreme that minimizes the mean-square error, which is the optimal weight. The LMS thus, approaches towards these optimal weights by ascending/descending down the mean-square-error vs filter weight curve.

The LMS algorithm for $p^{\text {th }}$ order algorithm can be summarized as

Parameters: $\quad p$ is the filter order,

$\mu$ is the step size

Initialization: $\quad \hat{h}(0)=0$

Computation: $\quad$ For $\mathrm{n}=0,1,2, \ldots$

$$
\begin{aligned}
& x(n)=[x(n), x(n-1), \ldots, x(n-p+1)]^{T} \\
& e(n)=d(n)-\hat{h}^{H}(n) x(n) \\
& \hat{h}(n+1)=\hat{h}(n)+\mu e^{*}(n) x(n)
\end{aligned}
$$

\subsection{Convergence and stability in the mean}

As the LMS algorithm does not use the exact values of the expectations, the weights would never reach the optimal weights in the absolute sense, but a convergence is possible in mean. That is even-though, the weights may change by small amounts, it changes about the optimal weights. However, if the variance with which the weights change, is large, convergence in mean would be misleading. This problem may occur, if the value of step-size $\mu$ is not chosen properly.

If $\mu$ is chosen to be large, the amount with which the weights change depends heavily on the gradient estimate, and so the weights may change by a large value so that gradient which was negative at the first instant may now become positive. And at the second instant, the weight may change in the opposite direction by a large amount because of the negative gradient and would thus keep oscillating with a large variance about the optimal weights. On the other hand if $\mu$ is chosen to be too small, time to converge to the optimal weights will be too

large. Thus, an upper bound on $\mu$ is needed which is given as $0<\mu<2 / \lambda_{\text {max }}$

where $\lambda_{\max }$ is the greatest eigenvalue of the autocorrelation matrix $R=E\{x(n) x(n)\}$. If this condition is not fulfilled, the algorithm becomes unstable and $\hat{h}(n)$ diverges.

Maximum convergence speed is achieved when $\mu=\frac{2}{\lambda_{\max }+\lambda_{\min }}$, where $\lambda_{\min }$ is the smallest eigenvalue of $\mathrm{R}$. Given that $\mu$ is less than or equal to this optimum, the convergence speed is determined by $\lambda_{\min }$, with a larger value yielding faster convergence. This means that faster convergence can be achieved when $\lambda_{\max }$ is close to $\lambda_{\min }$, that is, the maximum achievable convergence speed depends on the eigenvalue spread of R. A white noise signal has autocorrelation matrix $R=\sigma^{2} I$ where $\sigma^{2}$ is the variance of the signal. In this case all eigenvalues are equal, and the eigenvalue spread is the minimum over all possible matrices. The common interpretation of this result is therefore that the LMS converges quickly for white input signals, and slowly for colored input signals, such as processes with low-pass or high-pass characteristics.

It is important to note that the above upper-bound on $\mu$ only enforces stability in the mean, but the coefficients of $\hat{h}(n)$ can still grow infinitely large, i.e. divergence of the coefficients is still possible. A more practical bound is $0<\mu<2 / \operatorname{tr}[R]$, where $\operatorname{tr}[\mathrm{R}]$ denotes the trace of $\mathrm{R}$. This bound guarantees that the coefficients of $\hat{h}(n)$ do not diverge (in practice, the value of $\mu$ should not be chosen close to this upper bound, since it is somewhat optimistic due to approximations and assumptions made in the derivation of the bound). 


\section{NORMALIZED LEAST MEAN SQUARES FILTER (NLMS)}

The main drawback of the "pure" LMS algorithm is that it is sensitive to the scaling of its input $x(n)$. This makes it very hard (if not impossible) to choose a learning rate $\mu$ that guarantees stability of the algorithm. The Normalized least mean squares filter (NLMS) is a variant of the LMS algorithm that solves this problem by normalizing with the power of the input. The NLMS algorithm can be summarized as:

Parameters: $\quad p-$ filter order

$$
\mu-\text { step size }
$$

Initialization: $\hat{h}(0)=0$

Computation: For $\mathrm{n}=0,1,2, \ldots$

$$
\begin{aligned}
& x(n)=[x(n), x(n-1), \ldots, x(n-p+1)]^{T} \\
& e(n)=d(n)-\hat{h}^{H}(n) x(n) \\
& \hat{h}(n+1)=\hat{h}(n)+\frac{\mu e^{*}(n) x(n)}{x^{H}(n) x(n)}
\end{aligned}
$$

\subsection{Optimal learning rate}

It can be shown that if there is no interference $(v(n)=0)$, then the optimal learning rate for the NLMS algorithm is $\mu_{o p t}=1$ and is independent of the input $\mathrm{x}(\mathrm{n})$ and the real (unknown) impulse response $\mathrm{h}(\mathrm{n})$. In the general case with interference $(v(n) \neq 0)$ the optimal learning rate is

$$
\mu_{\text {opt }}=\frac{E\left[|y(n)-\hat{y}(n)|^{2}\right]}{E\left[|e(n)|^{2}\right]}
$$

The results above assume that the signals $v(n)$ and $\mathrm{x}(\mathrm{n})$ are uncorrelated to each other, which is generally the case in practice.

\section{BINARY STEP SizE AlgORITHMS}

\subsection{Binary Step Size LMS (BSSLMS)}

Here, we have two step sizes calculated from 2 values, delta and deviation. When the error increases from the previous value of error step size is delta+deviation. And when the error decreases from its previous value step size is delta-deviation. It has been found that this converges nearly as fast as NLMS algorithm.

\subsection{Binary Step Size NLMS (BSSNLMS)}

Here, we have two step sizes calculated from a single value delta. When the error increases from the previous value of error step size is delta+0.01. And when the error decreases from its previous value step size is delta-0.01. It has been found that this converges nearly as fast as NLMS algorithm, plus an added advantage that the mean square error decreases as compared to NLMS.

\section{Simulation RESULTS}

The performance of proposed as well as LMS and NLMS are presented in this section. In general the mean square error has to reach zero as soon as possible. But in practice, because of random nature of various phenomena makes it difficult for the automated systems to serve the purpose. The basic algorithm in the adaptive filtering, the LMS, initializes the process of predicting the unknown system or channel. But because of same step size the LMS takes considerable amount of time as well as complexity, modification of LMS in different ways are proposed in the literature. The performance of LMS algorithm is shown in the part a figures 5, 6 and 7. In fig. 5, the Mean Square Error (MSE) is plotted with respect to number of iterations with inherently represents the time to converge. As can be observed, in the case of LMS, the maximum value of MSE is around 0.45 . The MSE is under 0.05 after 50 iterations. But even after 500 iterations also the error is not less than 0.01 . In the Normalized LMS algorithm on the other hand the MSE has a maximum value around 0.85 .

The MSE is greater than 0.05 even till 100 iterations. From that point onwards the MSE is less than even 0.01. In the case BSSLMS, the maximum value of MSE is around 0.45. The MSE is less than 0.05 by 25 iterations itself. From 50 iterations onwards the MSE is less than 0.01. In the case BSSLMS, the maximum value of MSE is around 0.38. The MSE is greater than 0.1 even till 50 iterations. From 100 iterations onwards 
the MSE is less than 0.005 .
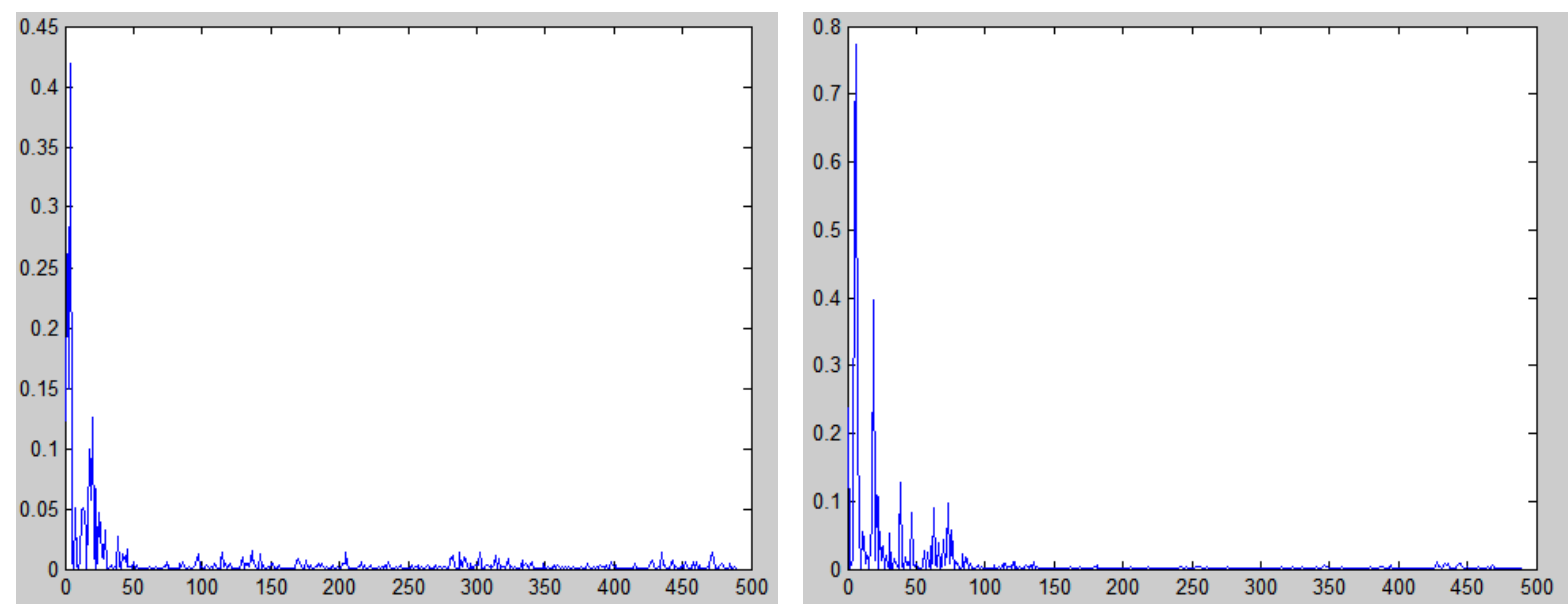

a) LMS

b) NLMS
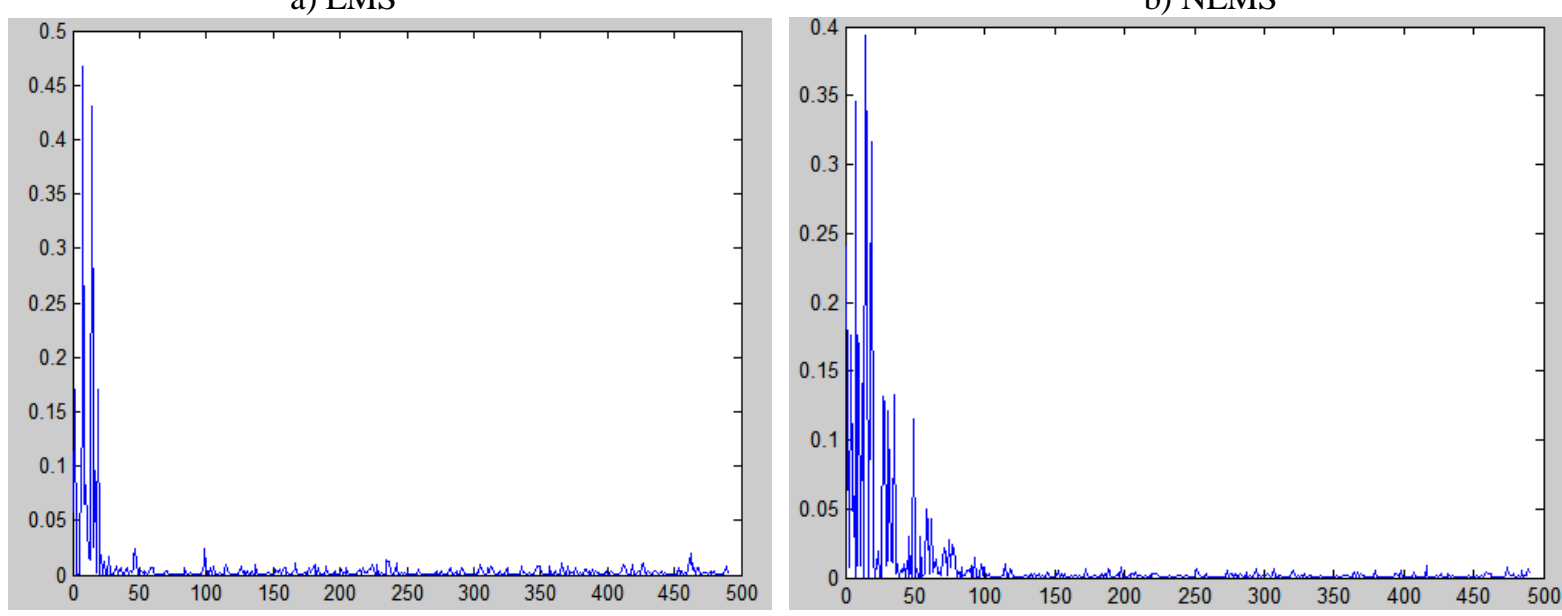

c) BSSLMS

d) BSSNLMS

Figure 4. Performace of Different Algorithms (MSE Vs No. Iterations(0-500)
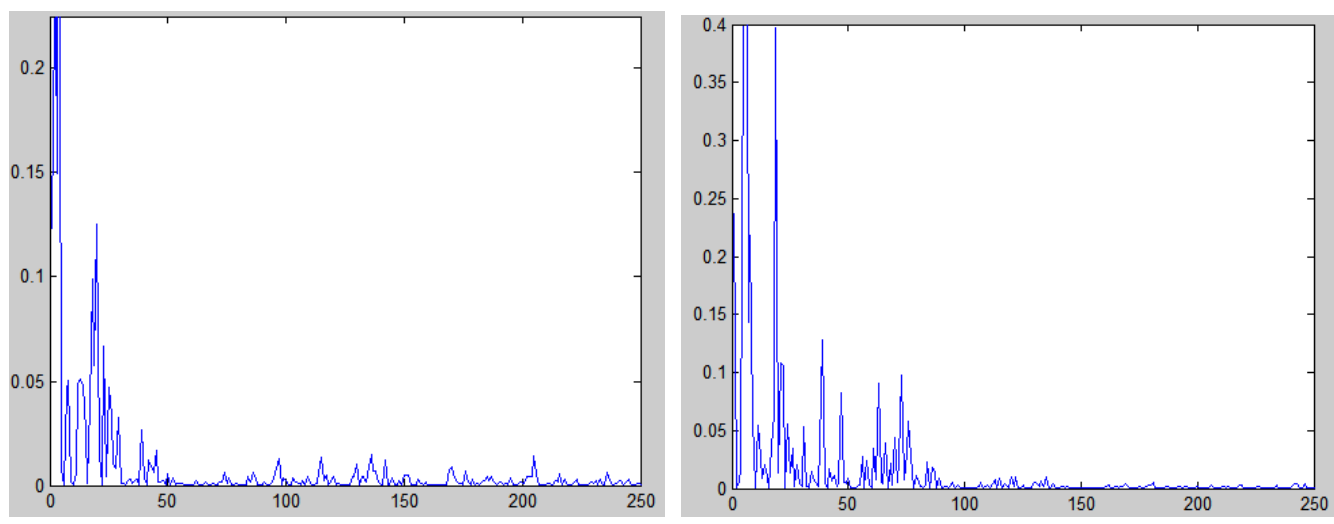

a) LMS

b) NLMS 


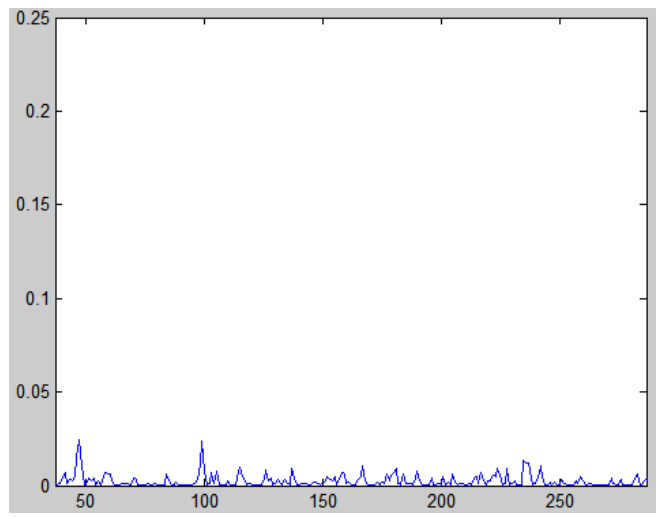

c) BSSLMS

Figure 5. Perfo

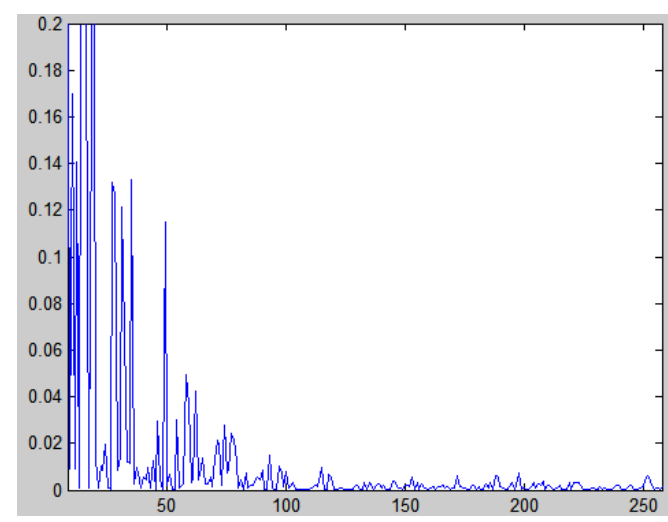

d) BSSNLMS

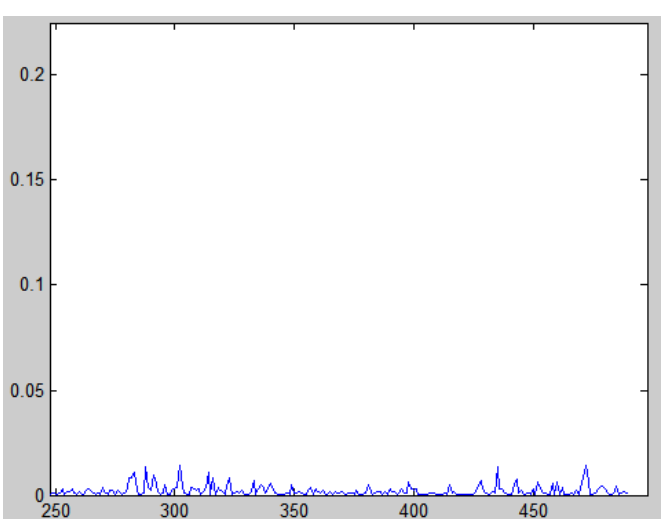

a) LMS

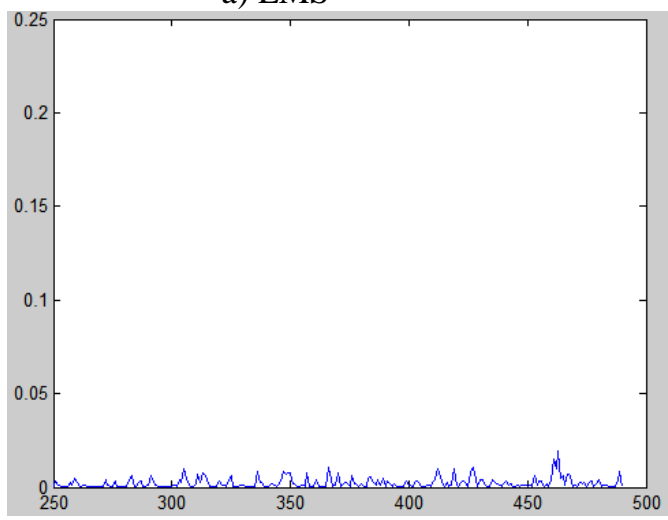

c) BSSLMS

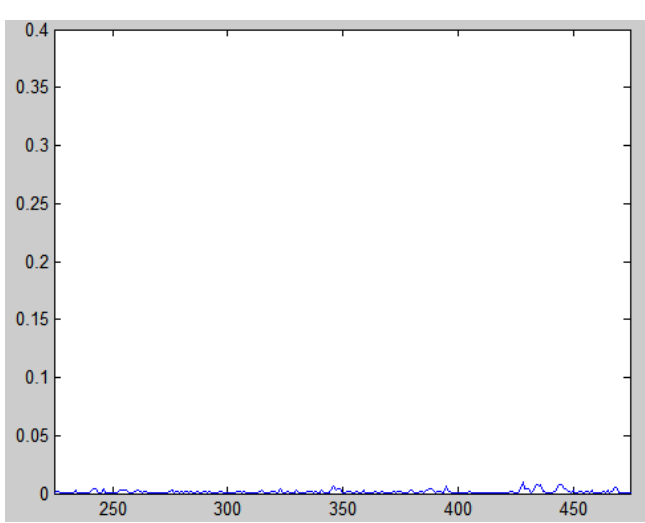

b) NLMS

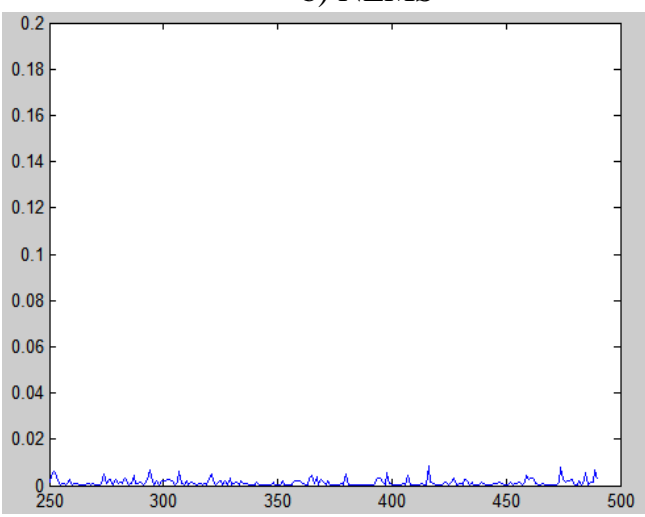

d) BSSNLMS

Figure 6. Performace of Different Algorithms (MSE Vs No. Iterations(250-500)

\section{CONCLUSIONS}

The proposed two algorithms are producing comparatively good results when compared with LMS and NLMS. The BSSLMS has excellent delay in the MSE in the first 25 iterations by that the average MSE, if we consider, would be very less compared to all the algorithms of our interest. The BSSNLMS on the other hand, has high MSE in the initial iterations but after 100 iterations the MSE almost 0.005 and it is still reducing in the further iterations. By that we can conclude that the proposed algorithms outperform the well-known LMS and NLMS. The obtained are comparable to those of [11]-[13].

\section{Journal Papers:}

\section{REFERENCES}

[1] W.-P. Ang, B. Farhang-Boroujeny, “A new class of gradient adaptive step-size LMS algorithms”. IEEE Trans. Signal Process. 49, $805-810$ (2001).

[2] C. Gazor, "Predictions in LMS-type adaptive algorithm for smoothly time-varying environments". IEEE Trans. Signal Process. 47, 1735-1739 (1999).

[3] D.G. Manolakis, V.K. Ingle, M.S. Kogan, "Statistical and Adaptive Signal Processing”, McGraw-Hill, New York, 2000. 
[4] L.C. Godara, “Applications of array antenna to mobile communications, Part I: Performance improvement, feasibility, and system considerations". Proc. IEEE 85, 1031-1060 (1997).

[5] L.C. Godara, "Applications of array antenna to mobile communications, Part II: Beam forming and direction-of-arrival considerations". Proc. IEEE 85, 1195-1238 (1997).

[6] S. Haykin, “Adaptive Filter Theory”, Prentice Hall, New York, 1995.

[7] J.H. Winters, "Signal acquisition and tracking with adaptive arrays in the digital mobile radio system IS-54 with flat fading". IEEE Trans. Veh. Technol. 42, 377-384 (1993).

[8] J.H. Winters, "Smart antenna for wireless systems". IEEE Pers. Commun. 23-27 (1998).

[9] V. Solo, X. Kong, “Adaptive Signal Processing Algorithms: Stability and Performance”, Prentice Hall, New York, 1995.

[10] Bernard Widrow and Marcian E. Hoff, “Adaptive Switching Circuits”, IRE Wescon Convention Record, 1960.

[11] Qi Zhang, Yuancheng Yao, Mingwei Qin, “An Uncorrelated Variable Step-size LMS Adaptive Algorithm”, Journal of Emerging Trends in Computing and Information Sciences, VOL. 3, NO.11 Nov, 2012.

[12] Mohammad purbagher, Abdolkhalegh Mohammadi, Javad Nourinia, Changiz ghobadi, “ Variable Step Variable Weights Lms (Vsvwlms) Algorithm By Modified Leaky Lms” International Journal of Modern Engineering Research (IJMER), Vol.2, Issue.4, July-Aug 2012 pp-2889-2892.

[13] Zhang Jingjing, "Variable Step Size LMS Algorithm", International Journal of Future Computer and Communication, Vol. 1, No. 4, December 2012.

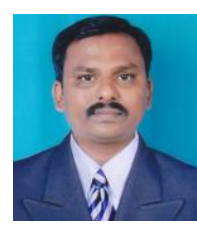

C. Mohan Rao (India) born on 21st April 1975, is pursuing Ph.D in Jawaharlal Nehru Technological University Anantapur. He received Master of Technology from Pondicherry Central University in the Year 1999, Bachelor of Technology form S.V University, Tirupati in the year 1997. He started his carrier as hardware Engineer in Hi-com technologies, during 1999 to 2001, after he worked as Assistant Professor in G. P. R. E. C during 2001 to 2007 and as Associate Professor in N. B. K. R. I. S. T. from 2007 to till date. $\mathrm{He}$ is a member of IETE and ISTE.

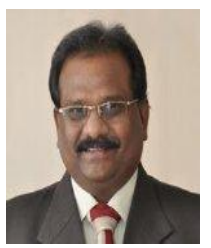

Dr. B. Stephen Charles (India) born on the 9th of August 1965. He received Ph.d degree in Electronics \& Communication Engineering from Jawaharlal Nehru Technological University, Hyderabad in 2001. His area of interest is Digital signal Processing. He received his B.Tech degree in Electronics and Communication Engineering from Nagarjuna University, India in 1986. He started his carrier as Assistant professor in Karunya institute of technology during 1989 to 1993, later joined as Associate Professor in K. S. R. M. College of Engg. During 1993 to 2001 after that he worked as Principal of St. John's College of Engineering \& Technology during 2001 to 2007 and now he is the Secretary, Correspondent and Principal in Stanley Stephen College of Engineering \& Technology, Kurnool. He has 24 years of teaching and research experience. He published more than 40 research papers in national and international journals and more than 30 research papers in national and international conferences. He is a member of Institute of Engineers and ISTE.

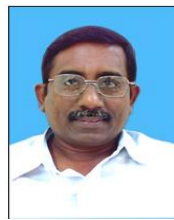

Dr. M.N. Giri Prasad received his B.Tech degree from J.N.T University College of Engineering, Anantapur, Andhrapradesh, India in 1982. M.Tech degree from Sri Venkateshwara University, Tirupati, Andhra Pradesh, India in 1994 and Ph.D degree from J.N.T. University, Hyderabad, Andhra Pradesh, Indian in 2003. Presently he is working as a Professor in the Department of Electronics and Communication at J.N.T University College of Engineering Anantapur, Andhrapradesh, India. He has more than 25 years of teaching and research experience. He has published more than 50 papers in national and international journals and more than 30 research papers in national and international conferences. His research areas are Wireless Communications and Biomedical instrumentation, digital signal processing, VHDL coding and evolutionary computing. He is a member of ISTE, IE \& NAFEN. 